\title{
Transgenic Expression of Laminin $\alpha 1$ Chain Does Not Prevent Muscle Disease in the $m d x$ Mouse Model for Duchenne Muscular Dystrophy
}

Kinga I. Gawlik, Bruno M. Oliveira, and Madeleine Durbeej

From the Department of Experimental Medical Science, Muscle Biology Unit, University of Lund, Lund, Sweden

Duchenne muscular dystrophy (DMD) is a severe neuromuscular disorder, and one of the most frequently encountered, but one for which there is as yet no treatment. Laminin-111 protein therapy was recently shown to be a promising approach to prevent muscle disease in the $m d x$ mouse model of DMD. The present study demonstrated that transgenic expression of laminin $\alpha 1$ chain in $m d x$ animals, resulting in laminin-111 heterotrimer formation in $m d x$ muscle, does not improve the dystrophic phenotype. The $m d x$ mice overexpressing laminin-111 ( $m d x$ LMa1) display features of $m d x$ littermates: dystrophic pattern of muscle biopsy, elevated creatine kinase levels, reduced muscle strength, and decreased sarcolemmal integrity. Increased expression of integrin $\alpha 7$ is not beneficial for $m d x \operatorname{LM} \alpha 1$ muscle, and components of the dystrophin-glycoprotein complex are not restored at the sarcolemma on laminin-111 overexpression. In summary, further studies are needed to verify the functionality of laminin-111 protein therapy in DMD and to describe the molecular events resulting from this approach. (AmJ Pathol 2011, 178:1728-1737; DOI: 10.1016/j.ajpath.2010.12.030)

Duchenne muscular dystrophy (DMD) is a severe, inherited neuromuscular disorder and the most prevalent form of muscular dystrophy, occurring in 1 of 3500 male births. ${ }^{1}$ DMD patients experience progressive muscle wasting, with clinical onset at 2 to 5 years of age; they lose the ability to walk between ages 7 to 13 , and die in their 20s because of cardiopulmonary failure. ${ }^{2}$ DMD is caused by deletions and mutations in the dystrophin gene $(D M D)^{3}$ that lead to absence of the sarcolemmadocked cytoskeletal protein and reduction of the dystrophin-glycoprotein complex (DGC), which together provide a mechanical link between the cytoskeleton and extracellular matrix. ${ }^{4,5}$ The $m d x$ dystrophin-deficient mouse is the best characterized mouse model for $\mathrm{DMD},{ }^{6,7}$ although the progressive muscle wasting presents itself in a much milder form than in humans. ${ }^{7}$

Although the genetic defects underlying DMD were identified more than 20 years ago, ${ }^{3}$ there is still no effective treatment of this devastating neuromuscular disease. Recently, however, a remarkable protein therapy strategy in the $m d x$ mouse was undertaken by Rooney et al, ${ }^{8}$ who demonstrated that systemic injections of laminin-111 derived from the Engelbreth-Holm-Swarm (EHS) tumor could ameliorate dystrophic symptoms in the $m d x$ mouse. Additionally, such an approach facilitated myoblast transplantation in this mouse model. ${ }^{9}$

Laminin-211, an extracellular matrix protein consisting of $\alpha 2, \beta 1$, and $\gamma 1$ chains, is the major laminin isoform in skeletal muscle. The $\alpha 2$ subunit binds to $\alpha$-dystroglycan (a DGC component) and to integrin $\alpha 7 \beta 1 .{ }^{10}$ Laminin-111 (consisting of $\alpha 1, \beta 1$, and $\gamma 1$ chains) is not expressed in skeletal muscle, but it has been shown to functionally replace laminin-211 in laminin $\alpha 2$ chain-deficient muscular dystrophy upon transgenic overexpression in the neuromuscular system. ${ }^{11-13}$ Similarly to the laminin $\alpha 2$ subunit, the laminin $\alpha 1$ chain binds to $\alpha$-dystroglycan ${ }^{14}$ and to integrin $\alpha 7 \beta 1 .{ }^{15}$ Of note, integrin $\alpha 7$ is upregulated in the skeletal muscle in both DMD patients and $m d x$ mice. ${ }^{16}$ Furthermore, integrin $\alpha 7$ has been proposed to be an important modifier of dystrophic symptoms in mice and to have roles complementary to those of the DGC. 8 , 17-20

Data reported by Rooney et $\mathrm{al}^{8}$ indicate that laminin111 is a highly effective therapeutic agent in the $m d x$ mouse model of DMD and could have applications in human disease. These remarkable results show promise for patients, and therefore the potency of laminin protein

Supported in part by the Muscular Dystrophy Association, the Anna-Greta Crafoord Foundation for Rheumatological Research, the Swedish National Research Council (Vetenskapsrådet), and the Alfred Österlund Foundation.

Accepted for publication December 22, 2010.

Address reprint requests to Kinga Gawlik, Ph.D., University of Lund, Department of Experimental Medical Science, Muscle Biology Unit, BMC B12, 22184 Lund, Sweden. E-mail: kinga.gawlik@med.lu.se. 
therapy needs to be tested in additional preclinical tests involving different molecular approaches.

In the present study, we tested by transgenic means the ability of murine laminin-111 to prevent dystrophindeficient muscular dystrophy in mice. We generated $m d x \mathrm{LM} \alpha 1$ mice, dystrophin-deficient animals that significantly overexpress laminin $\alpha 1$ chain (ie, the $\alpha$ subunit of the laminin-111 heterotrimer) in skeletal muscle. Transgenic expression of laminin $\alpha 1$ chain resulted in laminin111 formation in $m d x L M \alpha 1$ skeletal muscle basement membranes. Despite the substantial deposition of laminin$111, m d x \mathrm{LM} \alpha 1$ animals exhibited the same dystrophic features as $m d x$ mice, with fiber size variability, central nucleation, necrosis, sarcolemmal damage, decreased grip strength, and elevated creatine kinase (CK) levels. The expression of integrin $\alpha 7$ was increased in $m d x\llcorner M \alpha 1$ muscle, but the components of the DGC were not restored at the sarcolemma. Taken together, the present results contradict those of Rooney et $\mathrm{al}^{8}$ and suggest that laminin protein therapy in $m d x$ mice requires further verification.

\section{Material and Methods}

\section{Transgenic Animals}

C57BL/10ScSn-Dmdmdx/J $(m d x)$ mice were obtained from the Jackson Laboratory (Bar Harbor, ME). The transgenic animals overexpressing laminin $\alpha 1$ chain under the control of $\beta$-actin promoter (LM $\alpha 1 \mathrm{TG}$ ) have been described previously. ${ }^{11}$ The $m d x$ females were bred with $\mathrm{LM} \alpha 1 \mathrm{TG}$ males. All males born were $m d x$. These were further genotyped for the presence of the transgene (mdxLM $\alpha 1$ mice), as described previously. ${ }^{11}$ Mice were maintained in animal facilities according to animal care guidelines. All mouse experimentation was approved by the local (Lund district) ethics committee.

\section{Histology and Immunofluorescence Microscopy}

Cryosections ( $8 \mu \mathrm{m}$ thick) of skeletal muscle (quadriceps femoris, gastrocnemius, soleus, tibialis anterior, triceps brachii, and diaphragm) from 8- to 10-week-old wild-type, $m d x, m d x \mathrm{LM} \alpha 1$, and $d y^{3 K} \mathrm{LM} \alpha 1 \mathrm{TG}$ mice ( $n=3$ per group) were either stained with hematoxylin and eosin or subjected to immunofluorescence analysis using the following antibodies: rat monoclonal mAb200 against LM $\alpha 1 \mathrm{LG} 4,{ }^{11}$ rabbit polyclonal 15277 against dystrophin (Abcam, Cambridge, UK), rat monoclonal $4 \mathrm{H} 8-2$ against laminin $\alpha 2$ chain (Alexis Biochemicals; Enzo Life Sciences, Plymouth Meeting, PA), rat monoclonal MTn15 against tenascin-C, ${ }^{11}$ rat monoclonal LT3 against laminin $\beta 1$ chain (Chemicon; Millipore, Temecula, CA), rabbit polyclonal 1083+ against laminin $\gamma 1$ chain (kindly provided by Dr. T. Sasaki), rabbit polyclonal U31 against integrin $\alpha 7 \mathrm{~B}$ subunit (kindly provided by Dr. U. Mayer), ${ }^{21,22}$ mouse monoclonal IIH6 against $\alpha$-dystroglycan (Upstate Biotechnology, Lake Placid, NY), rabbit polyclonal against $\beta$-dystroglycan, ${ }^{11}$ mouse monoclonal 5B1 against $\beta$-sarcoglycan (Novocastra, Newcastle upon Tyne, UK), and mouse monoclonal DRP3/20C5 against utrophin (Novocastra). Stainings were performed as described previously. ${ }^{11,22,23}$ In addition, muscles from 5-week-old $m d x$ and $m d x\llcorner M \alpha 1$ animals were subjected to hematoxylin and eosin staining.

The area corresponding to tenascin-C labeling was quantified from stitched photos of the entire area of the diaphragm cross-section, using ImageJ software, version 143u (NIH, Bethesda, MD); five $m d x$ and six $m d x L M \alpha 1$ animals were analyzed. Unpaired $t$-test was used for statistical analysis. $P<0.05$ was considered statistically different. GraphPad Prism software, version 2.01 (La Jolla, CA) was used for all statistical analyses.

\section{Quantification of Fiber Size Distribution and Central Nucleation}

Diaphragm and limb muscles from three $m d x$ and three $m d x \mathrm{LM} \alpha 1$ animals (8 to 10 weeks old) were analyzed. For central nucleation, the tibialis anterior, triceps brachii, and diaphragm muscles were examined. The entire area of each muscle cross-section was considered (which corresponds to at least 1746, 1389, and 1889 fibers for each muscle type, respectively). For limbs, muscles from both collateral limbs were used. Unpaired $t$-test was used for statistical analysis, with significance set at $P<0.05$. Minimal Feret's diameter of muscle fibers ${ }^{24}$ was measured for at least 1993 and 1330 fibers in diaphragm and triceps brachii muscle, respectively. ImageJ software, version $143 \mathrm{u}(\mathrm{NIH})$ was used for measurements. The $\chi^{2}$ test was calculated for fiber distribution comparison, with significance set at $P<0.05$. All distributions of fiber size compared by pairs were related to the genotype.

\section{Treadmill Exercise and Evans Blue Dye Uptake}

Eight- to 11-week-old $m d x(n=3)$ and $m d x \mathrm{LM} \alpha 1$ mice $(n=4)$ were exercised for 30 minutes on a treadmill Exer $6 \mathrm{M}$ (Columbus Instruments, Columbus, $\mathrm{OH}$ ) at a downhill angle of 15 degrees. During the first 2 minutes, the speed was gradually increased from $7 \mathrm{~m} / \mathrm{min}$ to $14-16 \mathrm{~m} / \mathrm{min}$. Within 30 minutes after completed exercise, the mice were injected intraperitoneally with Evans Blue dye (EBD; SigmaAldrich, St. Louis, MO) dissolved in sterile saline $(0.5 \mathrm{mg}$ $\mathrm{EBD} / 0.05 \mathrm{~mL}$ saline; $50 \mu \mathrm{L} / 10 \mathrm{~g}$ body weight). After approximately 16 hours, muscles (quadriceps femoris, tibialis anterior, posterior compartment of calf, and triceps brachii) were collected and quickly frozen in liquid nitrogen. Unexercised mice were injected with EBD and used as controls. Cryosections $(8 \mu \mathrm{m})$ of the muscles were fixed in ice-cold acetone at $-20^{\circ} \mathrm{C}$ for 10 minutes and then were stained with laminin $\gamma 1$ antibody. Under fluorescence microscopy analysis, the muscle fiber EBD uptake was visualized by red emission. Total muscle area and area of EBD-positive fibers (red staining) in each muscle were quantified using ImageJ software, version 143u $(\mathrm{NIH})$. Muscles from both collateral limbs were used for quantification. Mann-Whitney U-test was used for statistical analysis, with significance set at $P<$ 0.05 . 


\section{Grip Strength Analyses}

Forelimb grip strength was measured on a grip strength meter (Columbus Instruments). Wild-type $(n=8), m d x$ ( $n=13)$, and $m d x \operatorname{LM} \alpha 1(n=15)$ males, 2 to 3 months old, were analyzed. Mice were pulling the metal bar five times. The two lowest values were rejected, and the mean of the three remaining values was counted. One-way analysis of variance followed by Bonferroni's multiple comparison test was used for statistical analysis, with significance set at $P<0.05$.

\section{A}

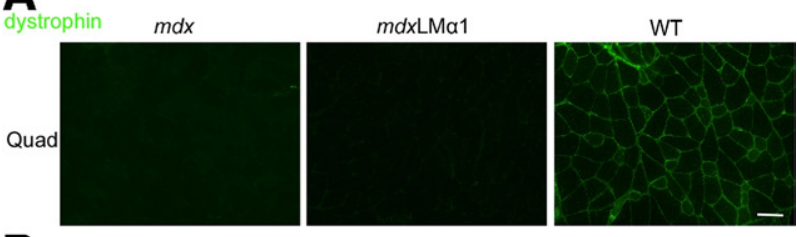

B
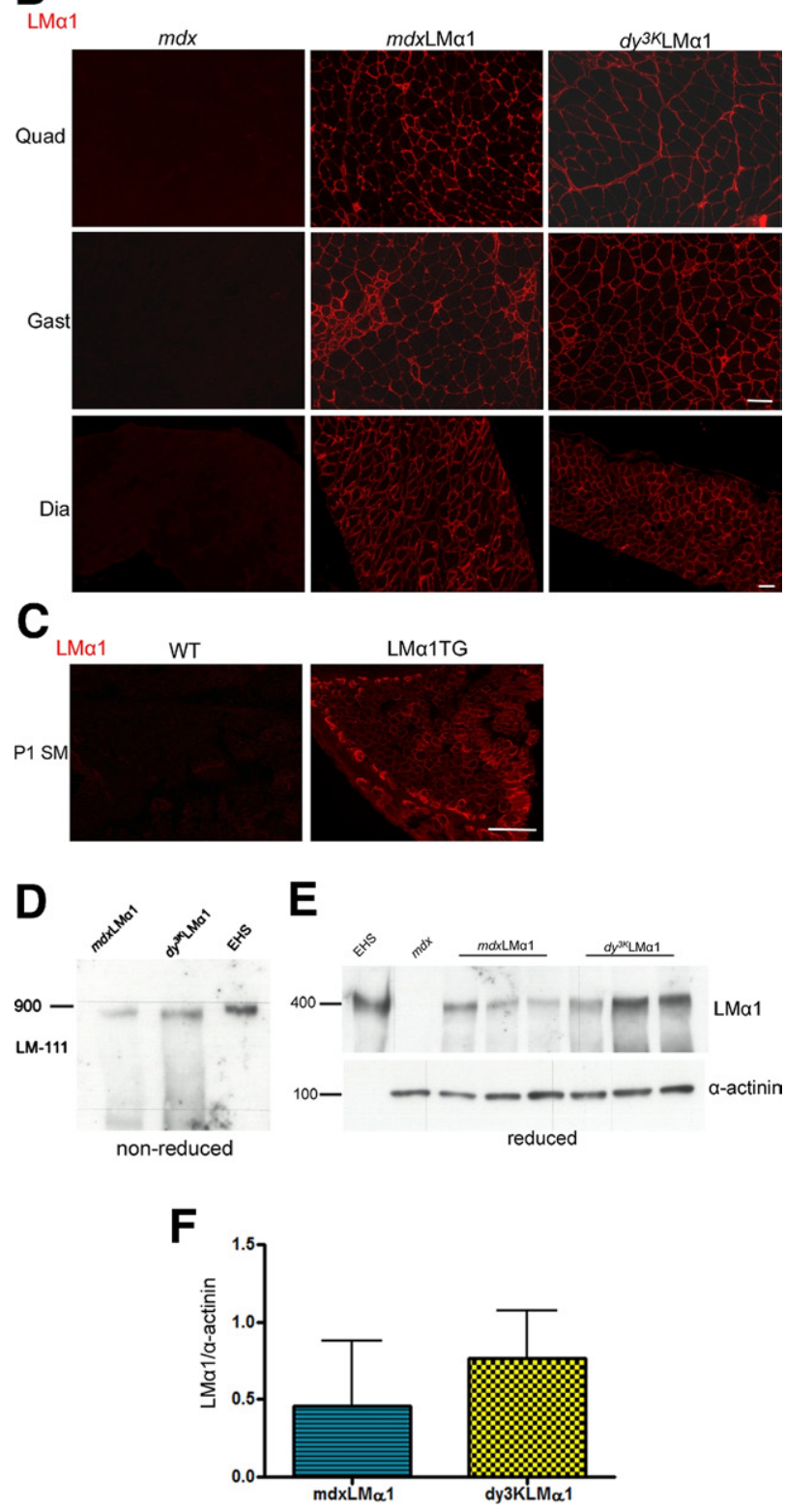

\section{Creatine Kinase Quantification}

Blood was collected from the tail vein of 2- to 3-month-old control $(m d x /+), m d x$, and $m d x \operatorname{LM} \alpha 1$ mice $(n=8 ; n=16$ and $n=16$, respectively) into EDTA tubes and was centrifuged two times for 5 minutes at $1100 \times$ g. Plasma was sent to the Clinical Chemistry Laboratory at Skåne University Hospital. The CK_P_S Cobas method was used to quantify enzyme activity. Kruskal-Wallis one-way analysis of variance followed by Dunn's test was used for statistical analysis, with significance set at $P<0.05$.

\section{Immunoblotting}

For laminin and integrin detection, proteins were isolated from $100 \mathrm{mg}$ of wild-type, $m d x, m d x \mathrm{LM} \alpha 1$, and $d y^{3 K} \mathrm{LM} \alpha 1$ muscles ( $n=3$ per group) as described previously. ${ }^{23}$ The supernatants were collected and the protein concentration was determined using a Pierce BCA assay (Thermo Fisher Scientific, Rockford, IL). The SDS-polyacrylamide gel electrophoresis and immunoblotting were performed as described previously. ${ }^{23}$ Integrin-containing samples were run under nonreducing conditions, and laminin-containing samples were run under both reducing and nonreducing conditions. Membranes were incubated overnight at $4^{\circ} \mathrm{C}$ with rabbit polyclonal antibody detecting laminin $\alpha 1$ chain, LG1-3 domains $(1: 500)^{23}$ (kindly provided by Dr. T. Sasaki); rabbit polyclonal antibody recognizing both laminin $\beta 1$ and $\gamma 1$ chain (1:1000; Sigma-Aldrich); and rabbit polyclonal antibody against integrin $\alpha 7 \mathrm{~B}$ (1:1500) (kindly provided by Dr. U. Mayer). Laminin $\alpha 1$ and $\beta 1 / \gamma 1$ chain expression was normalized to $\alpha$-actinin expression (detected with mouse monoclonal antibody DM1A, 1:3000; Sigma-Aldrich). One-way analysis of variance followed by Bonferroni's multiple comparison test was used for statistical analysis, except that for laminin $\alpha 1$ chain immunoblotting the Mann-Whitney $U$-test was applied, with significance set at $P<0.05$.

Figure 1. Laminin-111 expression in $m d x \mathrm{LM} \alpha 1$ mice. A: Dystrophin immunostaining in $m d x, m d x \mathrm{LM} \alpha 1$, and wild-type (WT) skeletal muscle confirms dystrophin absence from $m d x \mathrm{LM} \alpha 1$ muscle. B: Laminin $\alpha 1$ chain immunostaining demonstrates uniform expression of laminin $\alpha 1$ subunit in basement membranes of $m d x \mathrm{LM} \alpha 1$ skeletal muscle. It is expressed in a similar manner as in laminin $\alpha 2$ chain-deficient mice overexpressing laminin $\alpha 1$ chain ( $d y^{3 K} \mathrm{LM} \alpha 1$ ). As expected, it is absent from $m d x$ muscle. Quadriceps (Quad), gastrocnemius (Gast), and diaphragm (Dia) muscles are shown. C: Laminin $\alpha 1$ chain is not expressed in wild-type muscle of newborn mice at postnatal day 1 (P1), but is present in muscle from littermates overexpressing transgenic laminin $\alpha 1$ chain. Scale bars $=50 \mu \mathrm{m}(\mathbf{A}-\mathbf{C}$, all images $)$. D: Immunoblotting of skeletal muscle tissue extracts from $m d x \mathrm{LM} \alpha 1$ and $d y^{3 K} \mathrm{LM} \alpha 1$ mice and EHS laminin extract with a rabbit polyclonal antibody against laminin $\alpha 1$ LG3 domain under nonreducing conditions. The laminin-111 (LM-111) heterotrimer is present in $m d x \mathrm{LM} \alpha 1$ muscle (900-kDa band). E: Immunoblotting with the same antibody against laminin $\alpha 1$ chain and $\alpha$-actinin under reducing conditions. A 400-kDa band corresponding to laminin $\alpha 1$ chain is absent from $m d x$ skeletal muscle extract, but is present in $m d x \operatorname{LM} \alpha 1(n=3)$ and $d y^{3 K} \mathrm{LM} \alpha 1(n=3)$ muscles. EHS laminin was used as a positive control. F: Quantification of laminin $\alpha 1$ chain signals revealed no significant difference in expression between $m d x \operatorname{LM} \alpha 1$ and $d y^{3 K} \mathrm{LM} \alpha 1$ muscles $(P=0.4000)$ Laminin $\alpha 1$ chain expression was normalized to $\alpha$-actinin expression. MannWhitney $U$-test was used for statistical analysis, with significance set at $P<$ 0.05 . Results are reported as means $\pm \mathrm{SD}$. 


\section{Results}

\section{Generation of mdx Mice Overexpressing Laminin $\alpha 1$ Chain}

Transgenic mice overexpressing laminin $\alpha 1$ chain (LM $\alpha 1 \mathrm{TG}$ ) and producing laminin-111 in the neuromuscular system were shown to substantially alleviate congenital muscular dystrophy in laminin $\alpha 2$ chain-deficient mice. ${ }^{11-13}$ To test the ability of transgenically expressed laminin $\alpha 1$ chain to rescue the phenotype of $m d x$ mice, we crossed both strains to obtain dystrophin-deficient $m d x$ animals overexpressing laminin $\alpha 1$ subunit; these progeny are hereafter referred to as $m d x\llcorner M \alpha 1$ mice) (Figure 1, A and B). We next determined the expression of laminin $\alpha 1$ chain in $m d x\llcorner M \alpha 1$ skeletal muscle (Figure 1B). Laminin $\alpha 1$ chain is absent from basement membranes in mdx muscle tissue; however, immunofluorescence staining in $m d x \operatorname{LM} \alpha 1$ muscle showed continuous deposition of laminin $\alpha 1$ subunit alongside sarcolemma, in a similar manner as in laminin $\alpha 2$ chaindeficient mice overexpressing laminin $\alpha 1$ chain $\left(d y^{3 K} \mathrm{LM}_{\alpha 1} 1\right)$. Importantly, transgenic expression of laminin $\alpha 1$ in skeletal muscle is secured already at postnatal day 1 (Figure 1C) and even at embryonic stages (data not shown). We further investigated the expression of laminin $\alpha 1$ subunit by Western blot analyses. Similarly to $d y^{3 K} \mathrm{LM} \alpha 1$ muscle and EHS laminin extracts, laminin $\alpha 1$ chain is assembled into a laminin111 heterotrimer with laminin $\beta 1$ and $\gamma 1$ subunits (900 $\mathrm{kDa}$ ) in $m d x \mathrm{LM} \alpha 1$ skeletal muscle (analysis run under nonreduced conditions and probed with a laminin $\alpha 1$ chain antibody) (Figure 1D).

We also compared expression levels of laminin $\alpha 1$ subunit between $m d x \mathrm{LM} \alpha 1$ and $d y^{3 K} \mathrm{LM} \alpha 1$ muscle (Figure $1, \mathrm{E}$ and $\mathrm{F}$ ). Laminin $\alpha 1$ chain expression levels were not significantly different between two mouse strains, but there was a trend toward decreased production of laminin $\alpha 1$ subunit in $m d x\llcorner M \alpha 1$ muscle, compared with $d y^{3 K}$ LM $\alpha 1$ muscle (Figure $1, E$ and F). It is possible, however, that production of laminin $\alpha 1$ chain could be influenced by the normal expression of laminin $\alpha 2$ chain in $m d x \mathrm{LM} \alpha 1$ muscle (Figure 2A). Production of laminin $\alpha 2$ subunit also seemed unchanged in $m d x$ muscle (Figure 2A). Because laminin $\alpha$ chains can be secreted independently as monomers, ${ }^{25}$ we also analyzed whether sufficient amounts of laminin $\beta 1$ and $\gamma 1$ chains were available for the two major laminin $\alpha$ chains present in $m d x\llcorner M \alpha 1$ muscle basement membranes. Western blot analysis revealed substantial (5-fold) upregulation of laminin $\beta 1$ and $\gamma 1$ subunits in $m d x\llcorner M \alpha 1$ muscle, compared with wildtype and $m d x$ muscle (Figure 2, B and C). Immunofluorescent labeling with antibodies against laminin $\beta 1$ and $\gamma 1$ chains showed stronger signals in $m d x\llcorner M \alpha 1$ muscle and revealed the deposition of $\beta 1$ and $\gamma 1$ subunits in muscle basement membranes (Figure 2D), to which they are secreted together with laminin $\alpha 2$ and $\alpha 1$ chains (forming laminin-211 and laminin-111 heterotrimers, respectively).
A
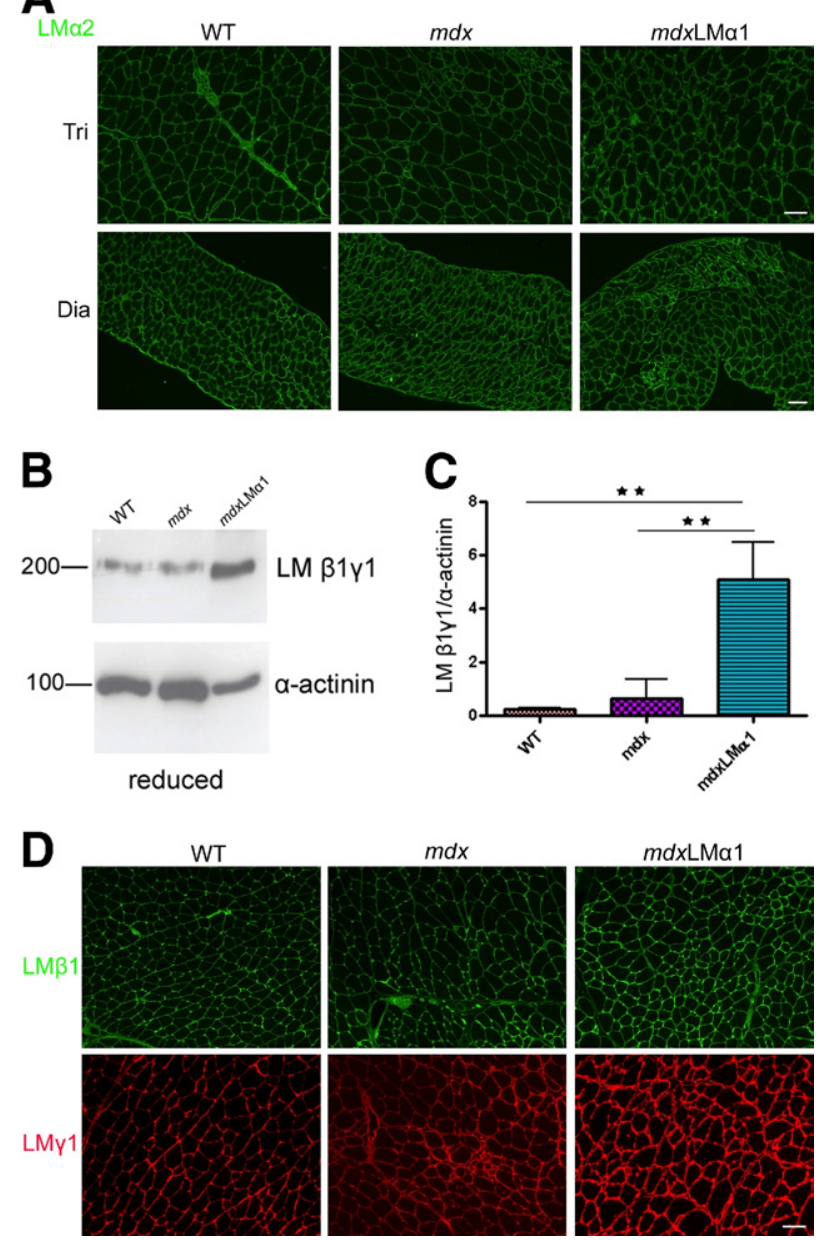

Figure 2. Expression of other laminin chains in $m d x \operatorname{LM} \alpha 1$ and $m d x$ mice A: Immunostaining with laminin $\alpha 2$ chain antibody reveals no reduction in laminin $\alpha 2$ chain expression in $m d x$ and $m d x \mathrm{LM} \alpha 1$ muscles, compared with the wild type. $\mathbf{B}$ and $\mathbf{C}$ : Immunoblotting of skeletal muscle tissue extracts from wild-type $(n=3), m d x(n=3)$, and $m d x \mathrm{LM} \alpha 1$ mice $(n=3)$ reveals significant 5-fold upregulation of laminin $\beta 1$ and $\gamma 1$ subunits (both at approximately $200 \mathrm{kDa}$ ) in $m d x \mathrm{LM} \alpha 1$ muscles, compared with both wild-type muscle $\left({ }^{* *} P<0001\right)$ and $m d x$ muscle $\left({ }^{* * *} P<0001\right)$. One-way analysis of variance followed by Bonferroni's multiple comparison test was used for statistical analysis. Results are reported as means \pm SD. D: Increased immunofluorescent signals for laminin $\beta 1$ and $\gamma 1$ chains in $m d x \operatorname{LM} \alpha 1$ muscle basement membranes, compared with wild-type and $m d x$ basement membranes. They form heterotrimers with $\alpha$ subunits. Scale bars: $50 \mu \mathrm{m}$ (A, all images in the same row, and $\mathbf{D}$, all images).

\section{The $\mathrm{mdxLM} \alpha 1$ Mice Are as Dystrophic as mdx Mice}

Despite high expression of laminin-111 in basement membranes of $m d x$ muscles, none of the dystrophic features were improved in $m d x \mathrm{LM} \alpha 1$ animals. The $m d x$ limb muscles undergo an early phase of degeneration with widespread necrosis, followed by a regenerative phase that is initiated at approximately week $6 .^{26}$ Diaphragm and limb muscles (quadriceps femoris, tibialis anterior, gastrocnemius, and triceps brachii) from 5-week-old mdx and $m d x \mathrm{LM} \alpha 1$ mice displayed similar extensive areas of acute necrosis (Figure 3) (tibialis anterior least affected). Analyses of 8 - to 10 -week-old $m d x$ and $m d x L M \alpha 1$ littermates revealed degenerating/regenerating and necrotic 


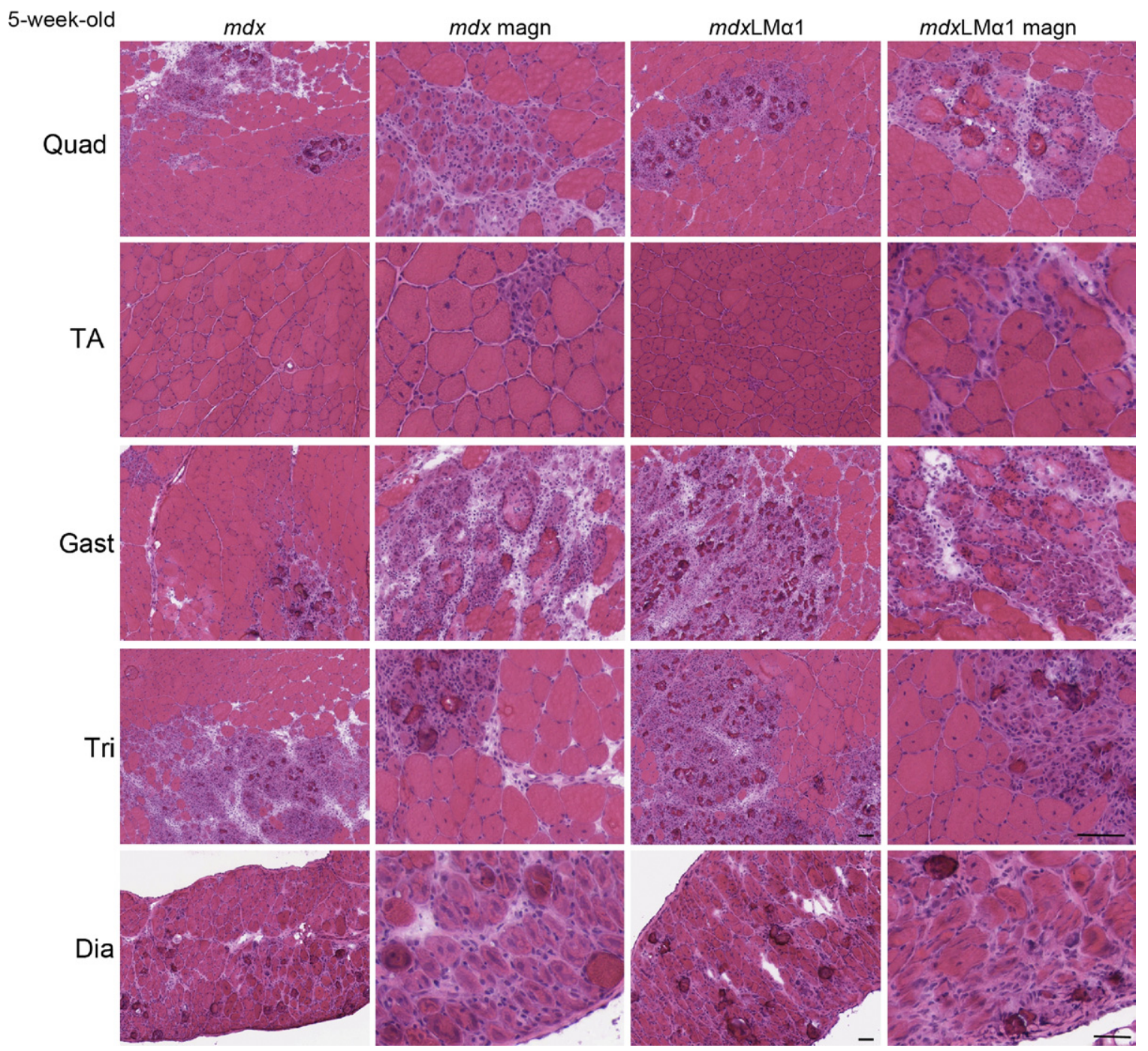

Figure 3. Histological analyses of 5-week-old $m d x$ and $m d x \mathrm{LM} \alpha 1$ muscles. Hematoxylin and eosin staining of quadriceps femoris (Quad), tibialis anterior (TA), gastrocnemius (Gast), triceps brachii (Tri), and diaphragm (Dia) muscles reveals dramatic focal necrosis in both $m d x$ and $m d x \mathrm{LM} \alpha 1$ muscles, often covering very large areas of muscle. The TA muscle was least affected by necrosis; only single, small necrotic patches were found. Scale bars: $50 \mu \mathrm{m}$.

regions with mononuclear cell infiltrates in all muscles (quadriceps, triceps brachii, soleus, and diaphragm are shown in Figure 4A; gastrocnemius and tibialis anterior are not shown). Fiber splitting and endomysial fibrosis (Figure 4A) were also evident, especially in diaphragm muscle. Detailed comparative analyses of central nucleation between $m d x$ and $m d x L M \alpha 1$ mice showed no significant difference in the amount of regenerating fibers (tibialis anterior, triceps brachii, and diaphragm), revealing robust muscle damage and degeneration/regeneration cycles in both genotypes (Figure 4B). Likewise, fiber size distribution was not significantly different between $m d x$ and $m d x\llcorner M \alpha 1$ diaphragm and triceps brachii muscles (Figure 4C).

Fibrosis is 10 times more pronounced in $\mathrm{mdx}$ diaphragm than in $m d x$ hindlimb muscles. ${ }^{27}$ We observed the same trend using antibodies against collagen III (data not shown) and tenascin-C. Hence, we quantified the expression of tenascin- $\mathrm{C}$ in diaphragm muscle from $\mathrm{mdx}$ and $m d x \mathrm{LM} \alpha 1$ mice (Figure 4D). Tenascin-C was depos- ited mostly focally in large patches in both genotypes, and fibrotic areas were not smaller in $m d x \mathrm{LM} \alpha 1$ diaphragm muscle (Figure 4D).

The $m d x$ mice are also characterized with contractioninduced damage of sarcolemma, ${ }^{28,29}$ decreased grip strength, ${ }^{30}$ and drastically elevated CK levels. ${ }^{31}$ Notably, sarcolemmal integrity was not increased in $\mathrm{mdx}$ mice upon laminin $\alpha 1$ chain overexpression. Nonexercised 2to 3-month-old $m d x \mathrm{LM} \alpha 1$ animals displayed substantial Evans Blue dye uptake in different muscles (Figure 5A). Treadmill exercise further enhanced muscle fiber damage in $m d x L M \alpha 1$, as well as in $m d x$ littermates (Figure $5 A$ ), and EBD-uptake did not differ significantly between the genotypes (Figure 5B).

Additionally, 2 - to 3-month-old $m d x \operatorname{LM} \alpha 1$ mice remained as weak as $m d x$ animals, as revealed by grip strength testing of forelimbs (Figure 5C). Finally, CK levels were substantially elevated in 2- to 3-month-old $m d x \mathrm{LM} \alpha 1$ mice, compared with control mice, and remained not significantly different from $m d x$ animals (Fig- 
A
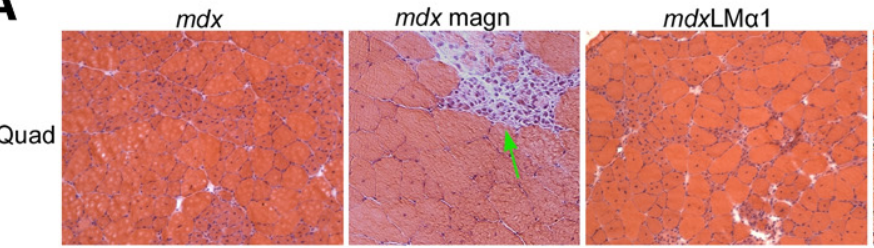

$m d x L M a 1$ magn
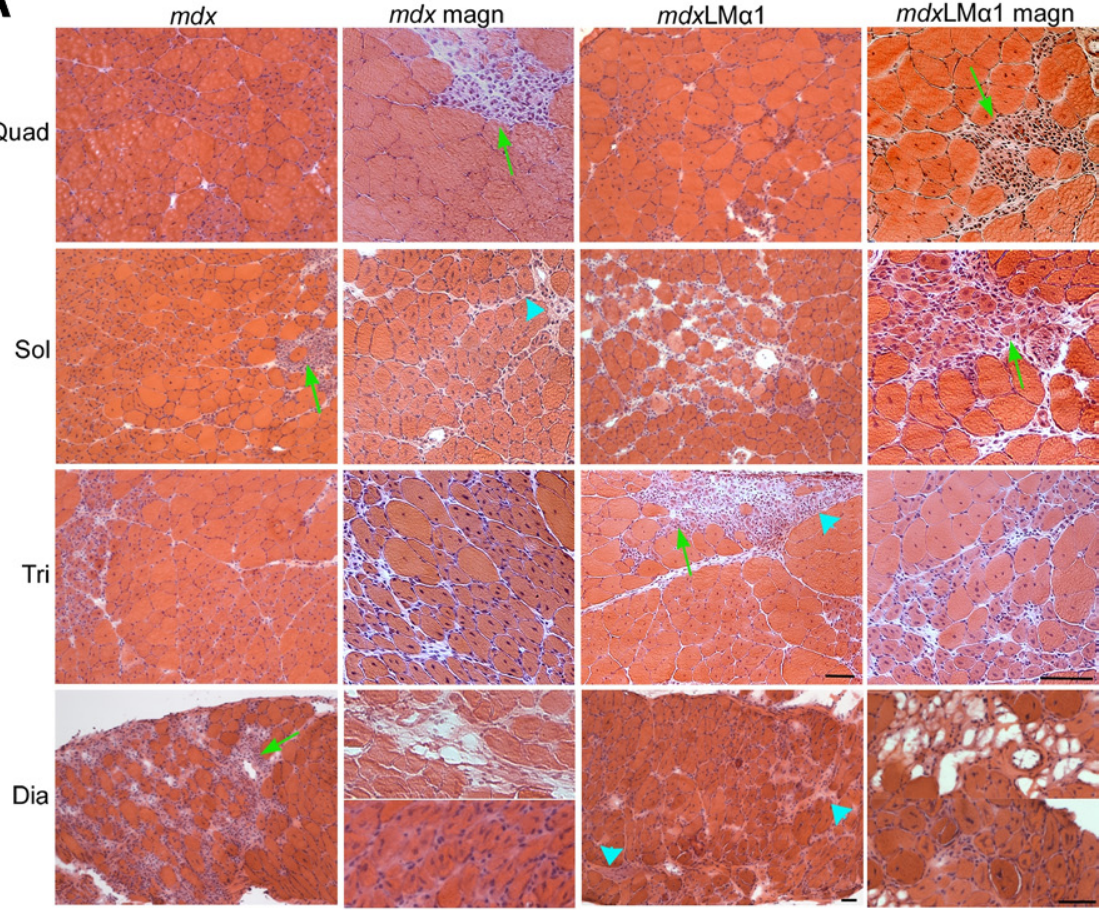

Dia

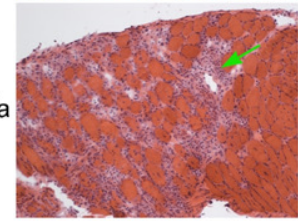

B
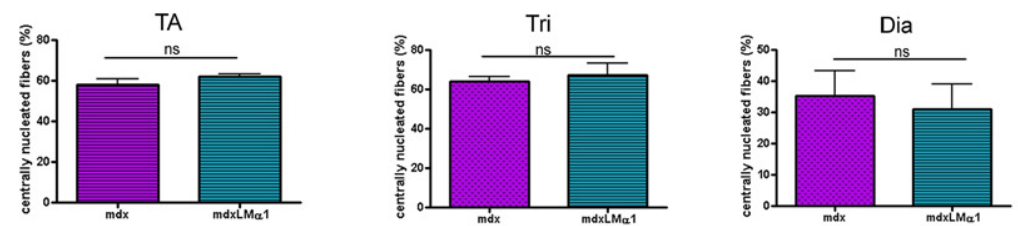

C
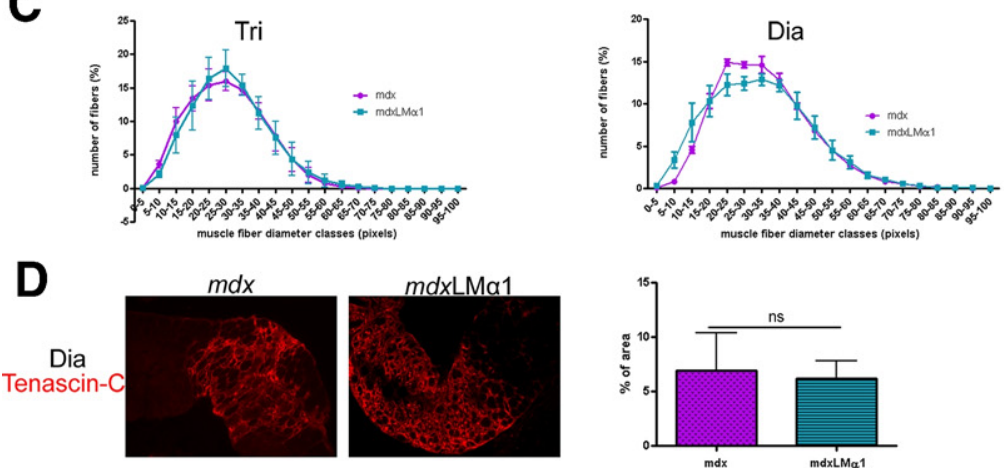

Figure 4. Analyses of $m d x \mathrm{LM} \alpha 1$ and $m d x$ muscle morphology. A: Hematoxylin and eosin staining of quadriceps femoris (Quad), soleus (Sol), triceps brachii (Tri), and diaphragm (Dia) muscles from 2-month-old $m d x \mathrm{LM} \alpha 1 \quad(n=3)$ and $m d x(n=3)$ mice reveals advanced muscular dystrophy in both genotypes. Images in columns 2 and 4 (magn) were taken at higher magnification than in columns 1 and 3 , and are from a different individual. Robust muscle degeneration/regeneration is evident as fibers contain centrally located nucleus. Necrotic areas and fibrotic lesions are indicated by arrows and arrowheads, respectively. The two separate magnified images for $m d x$ diaphragm muscle show presence of adipose tissue (top) and splitting fibers (bottom). B: Detailed analyses of centra nucleation of muscle fibers show no significant differences between $m d x(n=3)$ and $m d x \operatorname{LM} \alpha 1$ $(n=3)$ mice in all analyzed muscles (tibialis anterior, $P=0.0792$; triceps brachii, $P=0.4470$; and diaphragm, $P=0.5365$ ). Unpaired $t$-test was used for statistical analysis. Results are reported as means \pm SD. C: Fiber size distribution from $m d x \mathrm{LM} \alpha 1$ and $m d x$ triceps brachii $(n=3$ for both genotypes) and diaphragm muscle ( $n=3$ for both genotypes). There is no shift toward bigger muscle fibers in $m d x \mathrm{LM} \alpha 1$ muscles (triceps brachii, $P=0.9999$; diaphragm, $P=$ 0.9997). The $\chi^{2}$ test was used for statistical anal ysis. D: Tenascin-C labeling reveals fibrosis of diaphragm muscle from $m d x \mathrm{LM} \alpha 1$ animals ( $n=$ 6). The area of fibrotic lesions is not reduced, compared with diaphragm muscle from $m d x$ mice $(n=5)(P=0.7527)$. Unpaired $t$-test was used for statistical analysis. Results are shown as means \pm SD. Large patches of tenascin-C stained areas are shown in the micrographs, whereas the graphs represent the ratio of fibrotic lesion area to total area. The entire area of the diaphragm cross-section was used for quantification of tenascin-C staining. Scale bars: $50 \mu \mathrm{m}$. ure 5D), emphasizing the poor overall condition of $m d x \mathrm{LM} \alpha 1$ muscle and mirroring all of the dystrophic changes described here.

All these data confirm advanced muscular dystrophy in $m d x$ mice producing laminin-111 in skeletal muscle. Their dystrophic phenotype was not improved in any way, compared with $m d x$ littermates.

\section{Laminin $\alpha 1$ Chain Overexpression Additionally Increases the Expression of Integrin $\alpha 7$ but Does Not Restore DGC in the mdx Muscle}

It has been reported that expression of integrin $\alpha 7$ is increased in $m d x$ mice $e^{8,16}$ and in $m d x$ mice injected with laminin-111. ${ }^{8}$ In agreement with these reports, we detected an increase in expression of integrin $\alpha 7 \mathrm{~B}$ (the major cytoplasmic splice variant produced in muscle ${ }^{32}$ ) in $m d x$ and $m d x\llcorner M \alpha 1$ muscle, compared with wild-type muscle (Figure 6A). This was especially evident in limb muscles, and to a lesser extent in diaphragm muscle. Western blot analyses confirmed the upregulation of integrin $\alpha 7 \mathrm{~B}$ in $m d x$ animals ( 1.8 fold) and revealed further moderate increase in integrin $\alpha 7 \mathrm{~B}$ expression in $m d x \mathrm{LM} \alpha 1$ limb muscle, compared with $m d x$ limb muscle, to the same degree as detected by Rooney et $\mathrm{al}^{8}$ (1.4 fold) (Figure 6, B and C).

The injection of laminin-111 also resulted in enhanced expression of utrophin, ${ }^{8}$ which is the dystrophin homolog 
A

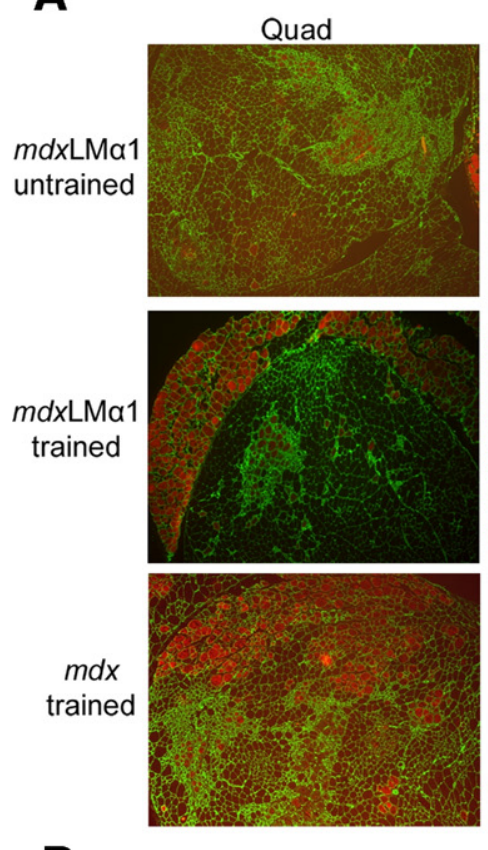

B

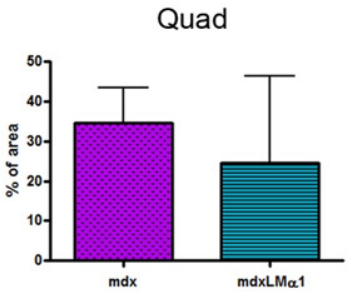

C grip strength

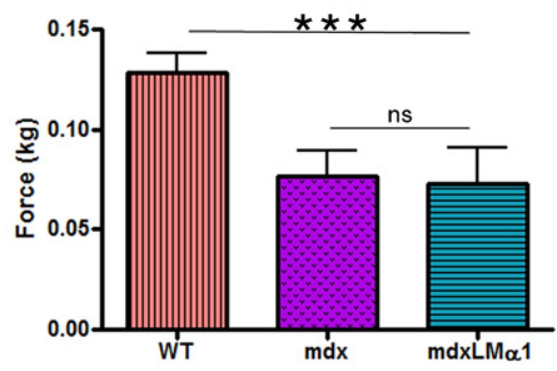

TA
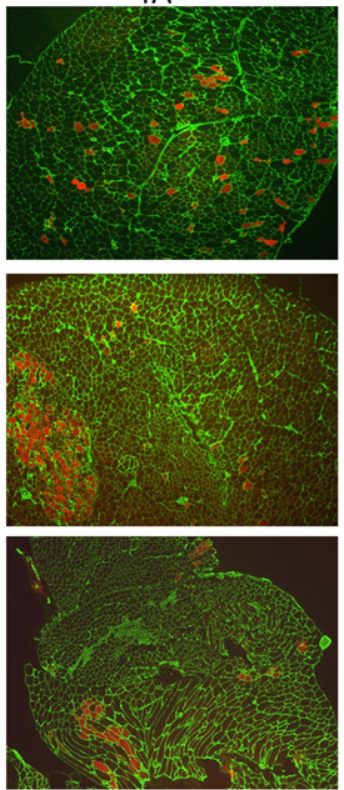

TA
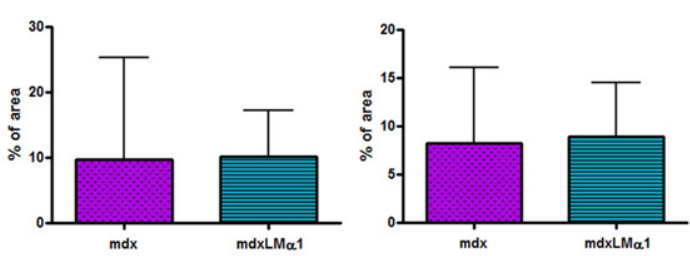

mdx
Calf
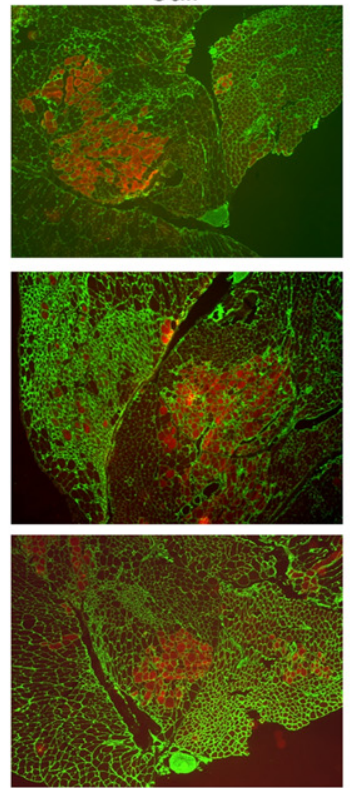

$\operatorname{Tr}$
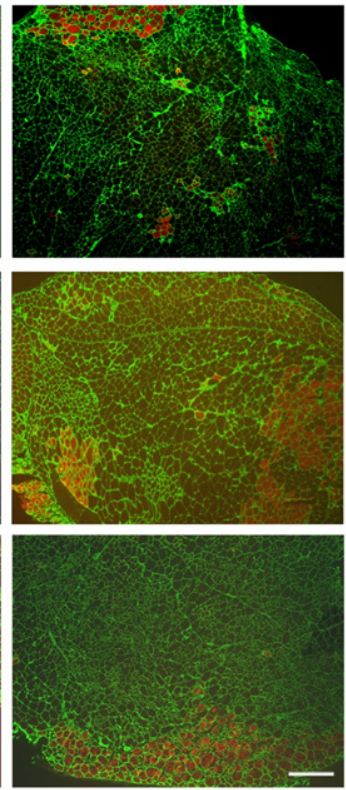

Tri

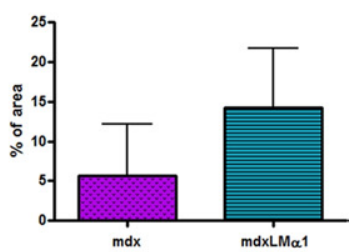

D $\mathrm{CK}$

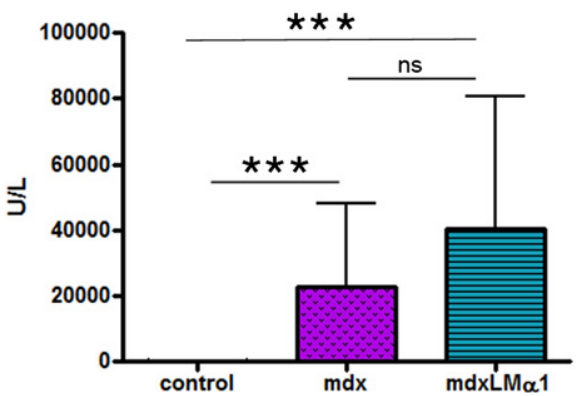

Figure 5. Examination of muscle function in $m d x \mathrm{LM} \alpha 1$ mice. A: Analyses of sarcolemmal integrity. Untrained $m d x \mathrm{LM} \alpha 1$ mice $(n=3)$ display damaged muscle fibers, as demonstrated by EBD uptake (fibers stained in red, top row). Treadmill exercise (trained) further enhances muscle injury in $m d x$ LM $\alpha 1$ mice $(n=4)$ (middle row). The $m d x$ mice subjected to training $(n=3)$ show a similar pattern of sarcolemmal disruption and EBD uptake as the $m d x$ LM $\alpha 1$ mice (bottom row). Quadriceps femoris (Quad), tibialis anterior (TA), calf, and triceps brachii (Tri) muscles from both legs were analyzed. Laminin $\gamma 1$ immunostaining (green) was used to covisualize muscle fibers. Moderately affected muscles were chosen for illustration. Scale bar $=100 \mu \mathrm{m}$ for all images. B: Quantification of EBD uptake in exercised $m d x$ and $m d x \mathrm{LM} \alpha 1$ mice. Sarcolemmal damage is not reduced in $m d x \mathrm{LM} \alpha 1$ muscles (quadriceps femoris, $P=0.4000 ;$ tibialis anterior $P=0.6286$ calf, $P=0.7213$; triceps brachii, $P=0.2286$ ). There is considerable variability of EBD uptake in $m d x$ and $m d x$ LM $\alpha 1$ mice between animals from the same group and even between opposing limbs from the same individual. Mann-Whitney $U$-test was used for statistical analysis. Results are reported as means \pm SD. C: Grip strength testing reveals no increase in forelimb muscle strength of $m d x \operatorname{LM} \alpha 1$ mice $(n=6)$, compared with $m d x$ animals $(n=7)(P>0.05)$. Both mouse genotypes remained significantly weaker than age-matched wild-type mice $(n=5)\left({ }^{* * * *} P<0.0001\right.$ for both $m d x$ and $m d x$ LM $\left.\alpha 1\right)$. One-way analysis of variance followed by Bonferroni's test was used for statistical analysis. Results are reported as means \pm SD. D: Serum CK activity in $m d x /+\operatorname{control}(n=8), m d x(n=10)$, and $m d x \mathrm{LM} \alpha 1$ $(n=7)$ mice. There was no difference in CK activity between $m d x$ and $m d x \mathrm{LM} \alpha 1$ animals $(P>0.05)$, but for both genotypes the CK level was significantly elevated compared with the $m d x$ / control (**** $P<0.0001$ for both $m d x$ and $m d x \mathrm{LM} \alpha 1$ ). Kruskal-Wallis one-way analysis of variance followed by Dunn's test was used for statistical analysis. Results are reported as means \pm SD. 
A

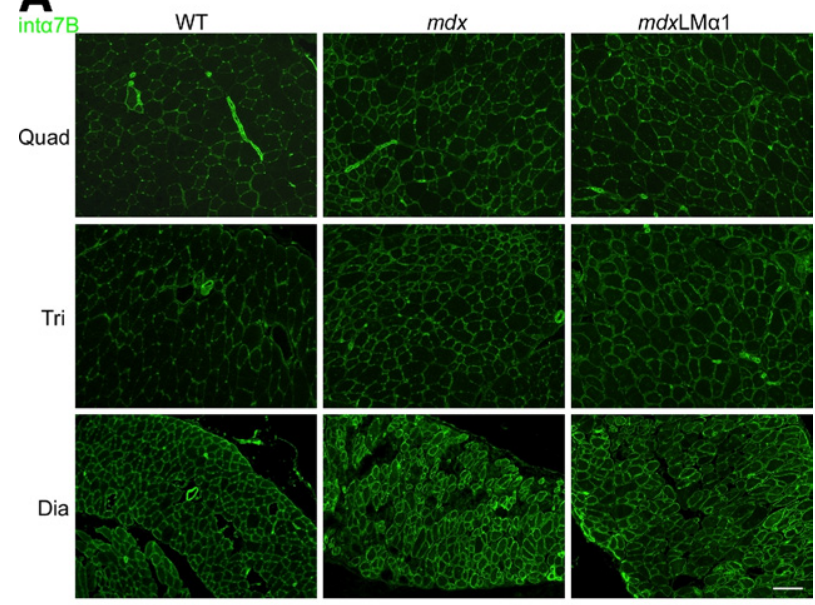

B

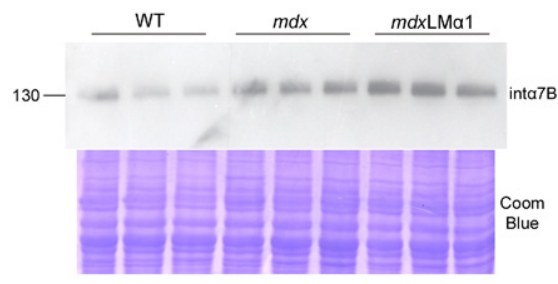

non-reduced

\section{C}

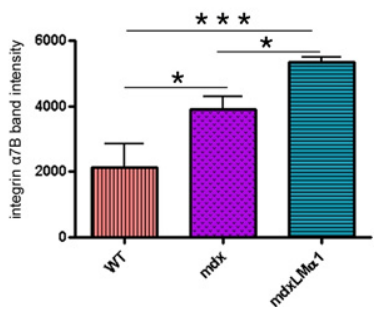

Figure 6. Analyses of integrin $\alpha 7 \mathrm{~B}$ expression in wild-type, $m d x$, and $m d x \mathrm{LM} \alpha 1$ mice. A: Immunostaining reveals upregulation of integrin $\alpha 7 \mathrm{~B}$ in $m d x$ and $m d x \mathrm{LM} \alpha 1$ muscles. Quadriceps, triceps brachii, and diaphragm are shown. Scale bar $=100 \mu \mathrm{m}$ for all images. B and C: Immunoblotting of skeletal muscle tissue extracts (nonreducing conditions) from wild-type ( $n=$ 3), $m d x(n=3)$, and $m d x \mathrm{LM} \alpha 1$ mice $(n=3)$ using integrin $\alpha 7 \mathrm{~B}$ antibody. Densitometric analysis confirms significant upregulation of integrin $\alpha 7 \mathrm{~B}$ in $m d x$ and $m d x \mathrm{LM} \alpha 1$ vs wild-type mice (1.8 fold, ${ }^{*} P<0.05 ; 2.5$-fold, ${ }^{* * * *} P<$ 0.0001 , respectively). Additionally, moderate increase of integrin $\alpha 7 \mathrm{~B}$ was noted in $m d x \mathrm{LM} \alpha 1$ limb muscles, compared with $m d x$ muscles (1.4 fold, $\left.{ }^{*} P<0.05\right)$. Coomassie Blue staining was shown to demonstrate equal loading. One-way analysis of variance followed by Bonferroni's multiple comparison test was used for statistical analysis. Results are reported as means \pm SD.

upregulated in $m d x$ animals. ${ }^{33}$ Additionally, utrophin was shown to functionally replace dystrophin upon transgenic overexpression in this mouse model. ${ }^{34}$ We therefore analyzed the expression of utrophin in $m d x$ mice overexpressing laminin $\alpha 1$ chain. Immunostaining revealed substantial upregulation of utrophin alongside sarcolemma in $m d x$ and $m d x\llcorner M \alpha 1$ limb muscles, compared with wildtype muscles, where it is expressed only in the neuromuscular junctions (Figure 7, arrows). The immunofluorescent signal did not differ appreciably between $m d x$ and $m d x \mathrm{LM} \alpha 1$ muscle; that a moderate utrophin upregulation upon laminin $\alpha 1$ chain overexpression cannot be distinguished by immunohistochemical means.
The effect of laminin-111 injections on expression of dystrophin-associated proteins is unknown, but laminin $\alpha 1$ chain overexpression could influence the expression of DGC components. We therefore analyzed the expression of $\alpha$-dystroglycan, $\beta$-dystroglycan, and $\beta$-sarcoglycan in wild-type, $m d x$, and $m d x \mathrm{LM} \alpha 1$ muscles. All three DGC components are severely reduced in $m d x$ muscle ${ }^{5}$ (Figure 7). Laminin-111 is a strong ligand for $\alpha$-dystroglycan, ${ }^{14}$ but none of the dystroglycan subunits (which link dystrophin/utrophin to the cell membrane and extracellular matrix) were restored at the sarcolemma upon laminin $\alpha 1$ chain overexpression in $m d x$ mice (Figure 7). Similarly, $\beta$-sarcoglycan expression was not normalized either (Figure 7). Lack of DGC restoration might be associated with failure of improvement of the dystrophic phenotype in $m d x\llcorner M \alpha 1$ mice, which further indicates that integrin $\alpha 7$ alone is not sufficient for amelioration of disease symptoms.

\section{Discussion}

To date, the laminin-111 protein therapy approach seems to bypass several major obstacles that hinder various genetic strategies for curing DMD. The results presented by Rooney et $\mathrm{al}^{8}$ are striking, considering that the injected $900-\mathrm{kDa}$ protein must traverse many barriers before it reaches skeletal muscle. It is debatable, however, whether laminin-111 would be beneficial for dystrophindeficient muscle, which already expresses normal levels of laminin-211, as well as elevated levels of integrin $\alpha 7$ and utrophin. Furthermore, it is remarkable that laminin111 could functionally replace dystrophin, a protein that

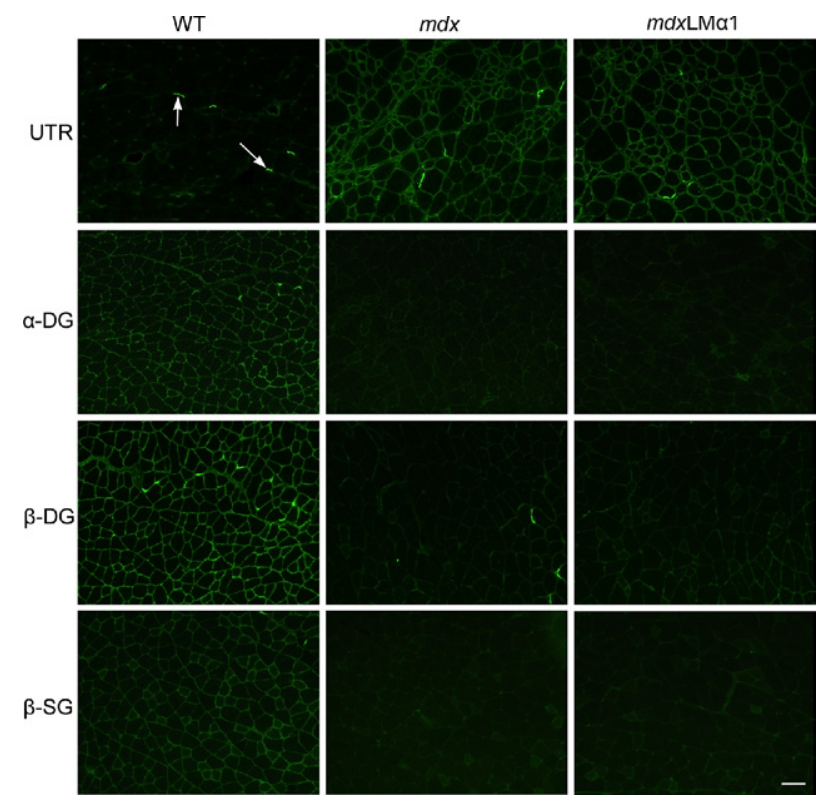

Figure 7. Expression of dystrophin-glycoprotein complex components in wild-type, $m d x$, and $m d x \mathrm{LM} \alpha 1$ limb muscles. In wild-type muscle, utrophin (UTR) is expressed at the neuromuscular junction (arrows). In $m d x$ and $m d x \operatorname{LM} \alpha 1$ muscle, it is abundantly present along the sarcolemma. On the other hand, $\alpha$-dystroglycan and $\beta$-dystroglycan (DG) and $\beta$-sarcoglycan (SG) are severely reduced in both $m d x$ and $m d x \mathrm{LM} \alpha 1$ muscle cell membranes. Scale bars: $100 \mu \mathrm{m}$ for all images. 
displays completely different structure, function, and subcellular localization. Hence, additional preclinical studies are needed to verify its functionality and safety and to describe the molecular events resulting from such intervention. In the present study, we tested by transgenic means the efficacy of laminin-111 in preventing dystrophin-deficient muscular dystrophy in mice. Although laminin $\alpha 1$ chain overexpression resulted in laminin-111 deposition in muscle basement membranes, its expression did not prevent the muscular dystrophy in $m d x$ animals.

Even allowing that our transgenic method is obviously different from the protein therapy introduced by Rooney et $a l^{8}{ }^{8}$ there is an evident discrepancy in the results obtained. One possibility is that injected EHS laminin undergoes fragmentation, which might facilitate the interaction of some laminin epitopes with their receptors. Hence, it is not excluded that smaller laminin molecules could be more effective in preventing the development of muscular dystrophy than a full-length laminin particle.

Another possible explanation for the discrepancies observed is that EHS laminin might be slightly different from endogenous ${ }^{35}$ or transgenic laminin-111, although it has also been shown that EHS laminin-111 is chemically, structurally, and immunologically identical to that obtained from nontumorigenic tissues. ${ }^{36}$ Additionally, commercial EHS laminin might contain other components that influence the outcome of laminin-111 protein therapy. Finally, although laminin $\alpha 1$ chain cDNA stays under the control of the $\beta$-actin promoter and in theory should be ubiquitously expressed, it is overexpressed primarily in the neuromuscular system. ${ }^{11,12}$ Injected EHS laminin might have a more systemic effect, and muscle phenotype improvement might result from global molecular events triggered by EHS laminin in different tissues.

Integrin $\alpha 7$ has been suggested to be one of the key molecules modifying disease progression in $m d x$ mice treated with EHS laminin. ${ }^{8}$ In our $m d x \mathrm{LM} \alpha 1$ mouse model, we also detected significant upregulation of integrin $\alpha 7 \mathrm{~B}$, but this event might not be sufficient to secure the reduction of dystrophic symptoms by laminin-111 in mdx mice. We have previously shown that laminin $\alpha 1$ chain overexpression regulates the expression of integrin $\alpha 7$ in laminin $\alpha 2$ chain-deficient mice, and that this positive regulation is beneficial for muscle tissue. ${ }^{22}$ In those animals, however, the entire DGC complex remains intact and available for interaction with laminin-111, certainly contributing to the rescue of dystrophic phenotype. This is further confirmed by our more recent data: when laminin-111 binding to integrin $\alpha 7$ is maintained, but simultaneously the link between laminin-111 and DGC is disrupted, limb muscles are not spared from laminin $\alpha 2$ chain-deficient muscular dystrophy. ${ }^{23}$ Thus, upregulation of integrin $\alpha 7$ alone, without concomitant complete restoration of DGC, might not lead to alleviation of the muscle phenotype in $m d x$ mice.

In summary, further steps are needed to verify the efficacy of laminin-111 injections into mice. Additionally, the molecular mechanisms triggered by systemic introduction of laminin-111 in muscle and other tissues must be characterized in more detail before bringing laminin111 protein therapy to clinical trials.

\section{Acknowledgments}

We thank Drs. Takako Sasaki and Ulrike Mayer for providing antibodies and Dr. Virginie Carmignac for help with $\chi^{2}$ testing.

\section{References}

1. van Deutekom JC, van Ommen GJ: Advances in Duchenne muscular gene therapy. Nat Rev Genet 2003, 10:774-783

2. Voit T, Tomé FMS. The congenital muscular dystrophies. Myology, ed 3. Edited by Engel AG, Franzini-Armstrong C. New York, McGraw-Hill, 2004, pp 1203-1238

3. Hoffman EP, Brown RH Jr, Kunkel LM: Dystrophin: the protein product of the Duchenne muscular dystrophy locus. Cell 1987, 51:919-928

4. Ervasti JM, Campbell KP: Membrane organization of the dystrophinglycoprotein complex. Cell 1991, 66:1121-1131

5. Ohlendieck K, Campbell KP: Dystrophin-associated proteins are greatly reduced in skeletal muscle from mdx mice. J Cell Biol 1991, 115:1685-1694

6. Sicinski P, Geng Y, Ryder-Cook AS, Barnard EA, Darlison MG, Barnard PJ: The molecular basis of muscular dystrophy in the $\mathrm{mdx}$ mouse: a point mutation. Science 1989, 244:1578-1580

7. Durbeej M, Campbell KP: Muscular dystrophies involving the dystrophin-glycoprotein complex: an overview of current mouse models. Curr Opin Genet Dev 2002, 12:349-361

8. Rooney JE, Gurpur PB, Burkin DJ: Laminin-111 protein therapy prevents muscle disease in the mdx mouse model for Duchenne muscular dystrophy [Erratum appeared in Proc Natl Acad Sci USA 2009 106:15514]. Proc Natl Acad Sci USA 2009, 106:7991-7996

9. Goudenege S, Lamarre Y, Dumont N, Rousseau J, Frenette J, Skuk D, Tremblay JP: Laminin-111: a potential therapeutic agent for Duchenne muscular dystrophy. Mol Ther 2010, 18:2155-2163

10. Sciandra F, Gawlik KI, Brancaccio A, Durbeej M: Dystroglycan, a possible mediator for reducing congenital muscular dystrophy? Trends Biotechnol 2007, 25:262-268

11. Gawlik K, Miyagoe-Suzuki Y, Ekblom P, Takeda S, Durbeej M: Laminin alpha1 chain reduces muscular dystrophy in laminin alpha2 chain deficient mice. Hum Mol Genet 2004, 13:1775-1784

12. Gawlik KI, Li JY, Petersén ÅA, Durbeej M: Laminin alpha1 chain improves laminin alpha2 chain deficient peripheral neuropathy. Hum Mol Genet 2006, 15:2690-2700

13. Gawlik K, Durbeej M: Transgenic overexpression of laminin alpha1 chain in laminin alpha2 chain deficient mice rescues the disease throughout the lifespan. Muscle Nerve 2010, 42:30-37

14. Talts JF, Andac Z, Göhring W, Brancaccio A, Timpl R: Binding of the $G$ domains of laminin alpha1 and alpha2 chains and perlecan to heparin, sulfatides, alpha-dystroglycan and several extracellular matrix proteins. EMBO J 1999, 18:863-870

15. von der Mark H, Williams I, Wendler O, Sorokin L, von der Mark K, Pöschl $\mathrm{E}$ : Alternative splice variants of alpha7beta1 integrin selectively recognize different laminin isoforms. J Biol Chem 2002, 277:6012-6016

16. Hodges BL, Hayashi YK, Nonaka I, Wang W, Arahata K, Kaufman SJ: Altered expression of the alpha7beta1 integrin in human and murine muscular dystrophies. J Cell Sci 1997, 110:2873-2881

17. Burkin DJ, Wallace GQ, Nicol KJ, Kaufman DJ, Kaufman SJ: Enhanced expression of the alpha7beta1 integrin reduces muscular dystrophy and restores viability in dystrophic mice. J Cell Biol 2001, 152:1207-1218

18. Burkin DJ, Wallace GQ, Milner DJ, Chaney EJ, Mulligan JA, Kaufman $\mathrm{SJ}$ : Transgenic expression of alpha7beta1 integrin maintains muscle integrity, increases regenerative capacity, promotes hypertrophy, and reduces cardiomyopathy in dystrophic mice. Am J Pathol 2005, 166:253-263

19. Guo C, Willem M, Werner A, Raivich G, Emerson M, Neyses L, Mayer $U$ : Absence of alpha7 integrin in dystrophin-deficient mice causes a myopathy similar to Duchenne muscular dystrophy. Hum Mol Genet 2006, 15:989-998 
20. Rooney JE, Welser JV, Dechert MA, Flintoff-Dye NL, Kaufman SJ, Burkin DJ: Severe muscular dystrophy in mice that lack dystrophin and alpha7 integrin. J Cell Sci 2006, 119:2185-2195

21. Cohn RD, Mayer U, Saher G, Herrmann R, van der Flier A, Sonnenberg A, Sorokin L, Voit T: Secondary reduction of alpha7B integrin in laminin alpha2 deficient congenital muscular dystrophy supports an additional transmembrane link in skeletal muscle. J Neurol Sci 1999, 163:140-152

22. Gawlik KI, Mayer U, Blomberg K, Sonnenberg A, Ekblom P, Durbeej M: Laminin alpha1 chain mediated reduction of laminin alpha2 chain muscular dystrophy involves integrin alpha7beta1 and dystroglycan. FEBS Lett 2006, 580:1759-1765

23. Gawlik KI, Åkerlund M, Carmignac V, Elamaa H, Durbeej M: Distinct roles for laminin globular domains in laminin alpha1 chain mediated rescue of murine laminin alpha2 chain deficiency. PLoS One 2010, 5:e11549

24. Briguet A, Courdier-Fruh I, Foster M, Meier T, Magyar JP: Histological parameters for the quantitative assessment of muscular dystrophy in the mdx-mouse. Neuromuscul Disord 2004, 14:675-682

25. Yurchenco PD, Quan Y, Colognato H, Mathus T, Harrison D, Yamada Y, O'Rear JJ: The alpha chain of laminin-1 is independently secreted and drives secretion of its beta- and gamma-chain partners [Erratum appeared in Proc Natl Acad Sci USA 1998, 95:1968]. Proc Natl Acad Sci USA 1997, 94:10189-10194

26. Coulton GR, Morgan JE, Partridge TA, Sloper JC: The mdx mouse skeletal muscle myopathy: I. A histological, morphometric and biochemical investigation. Neuropathol Appl Neurobiol 1988, 14: 53-70

27. Stedman HH, Sweeney HL, Shrager JB, Maguire HC, Panettieri RA, Petrof B, Narusawa M, Leferovich JM, Sladky JT, Kelly AM: The mdx mouse diaphragm reproduces the degenerative changes of Duchenne muscular dystrophy. Nature 1991, 352:536-539

28. Petrof BJ, Shrager JB, Stedman HH, Kelly AM, Sweeney HL: Dystrophin protects the sarcolemma from stresses developed during muscle contraction. Proc Natl Acad Sci USA 1993, 90:3710-3714

29. Straub V, Rafael JA, Chamberlain JS, Campbell KP: Animal models for muscular dystrophy show different patterns of sarcolemmal disruption. J Cell Biol 1997, 139:375-385

30. Connolly AM, Keeling RM, Mehta S, Pestronk A, Sanes JR: Three mouse models of muscular dystrophy: the natural history of strength and fatigue in dystrophin-, dystrophin/utrophin-, and laminin alpha2deficient mice. Neuromuscul Disord 2001, 11:703-712

31. Glesby MJ, Rosenmann E, Nylen EG, Wrogemann K: Serum CK, calcium, magnesium, and oxidative phosphorylation in $\mathrm{mdx}$ mouse muscular dystrophy. Muscle Nerve 1988, 11:852-856

32. Crawley S, Farrell EM, Wang W, Gu M, Huang HY, Huynh V, Hodges BL, Cooper DN, Kaufman SJ: The alpha7beta1 integrin mediates adhesion and migration of skeletal myoblasts on laminin. Exp Cell Res 1997, 235:274-286

33. Matsumura K, Ervasti JM, Ohlendieck K, Kahl SD, Campbell KP: Association of dystrophin-related protein with dystrophin-associated proteins in mdx mouse muscle. Nature 1992, 360:588-591

34. Tinsley J, Deconinck N, Fisher R, Kahn D, Phelps S, Gillis JM, Davies $\mathrm{K}$ : Expression of full-length utrophin prevents muscular dystrophy in mdx mice. Nat Med 1998, 12:1441-1444

35. Zolkiewska A, Thompson WC, Moss J: Interaction of integrin alpha7beta1 in C2C12 myotubes and in solution with laminin. Exp Cell Res 1998, 240:86-94

36. Kleinman HK: Isolation of laminin-1 and type IV collagen from EHS sarcoma. J Tissue Cult Methods 1994, 16:231-233 